\title{
CARACTERIZAÇÃO FÍSICA DE FILÉS DE SURUBIM (Pseudoplatystoma sp.), PACU (Piaractus mesopotamicus) E PIRARUCU (Arapaimas gigas)
}

\author{
Claucia Aparecida Honorato \\ Andreia Caneppele \\ Jéssica Carmo Matoso \\ Mirelli Ribeiro Prado \\ Mayara Schueroff Siqueira \\ Luana Rafaela Oliveira Souza
}

HONORATO, C. A.; CANEPPELE, A.; MATOSO, J. C.; PRADO, M. R.; SIQUEIRA, M. S.; SOUZA, L. R. O. Caracterização física de filés de Surubim (Pseudoplatystoma sp.), Pacu (Piaractus mesopotamicus) e Pirarucu (Arapaimas gigas). Arq. Ciênc. Vet. Zool. UNIPAR, Umuarama, v. 17, n. 4, p. 237-242, out./dez. 2014.

RESUMO: O objetivo deste estudo foi avaliar as características físicas de filé de surubim (Pseudoplatystoma sp.) pacu (Piaractus mesmopotamicus) e do pirarucu (Arapaimas gigas). Foram adquiridos 10 exemplares de surubim, pacu e postas de filé de pirarucu. Foram aferidos os parâmetros de rendimento de carcaça e as características físicas dos filés como a cor da carne, capacidade de retenção de água, força de cisalhamento e perda de líquido durante o cozimento. O rendimento de filé foi comprado entre as espécies surubim e pacu em delineamento inteiramente casualizado (DIC) com dois tratamentos e 10 repetições. As características físicas do filé utilizou-se o DIC com três tratamentos com dez repetições, comparadas pelo teste de Tukey. O rendimento de filé do surubim foi superior ao do pacu. As perdas de água após o descongelamento foram mais pronunciadas no pirarucu em comparação ao surubim. A capacidade de retenção de água das amostras de pirarucu foi significativamente superior as do surubim. O pH do filé de surubim foi de $6,18 \pm 0,08$ e do filé de pirarucu de $6,27 \pm 0,15$ e não apresentaram diferenças significativas. A coloração do filé do surubim e do pirarucu são muito semelhantes devido à qualidade da alimentação fornecida durante a produção em cativeiro dessas espécies de peixe. Os filés das espécies de peixe do estudo apresentam características de carne suculenta, macia e de coloração clara. Destaca-se a necessidade de realização de futuros testes de aceitabilidade e de processamento desta carne para estimular o consumo.

PALAVRAS-CHAVE: Pescado. Peixes nativos. File de peixe.

\section{PHYSICAL CHARACTERIZATION OF FILLETS OF SURUBI (Pseudoplatystoma sp.), PACU (Piaractus mesopo- tamicus) AND PIRARUCU (Arapaimas gigas)}

\begin{abstract}
The objective of this study is to evaluate the physical characteristics of fillets from surubi (Pseudoplatystoma sp.) pacu (Piaractus mesmopotamicus) and pirarucu (Arapaimas gigas). A total of 10 samples of surubi, pacu and pirarucu fillet slices was purchased. Carcass yield and fillet physical characteristic parameters were measured, including meat color, water-retention capacity, shearing strength and loss of liquid during cooking. Fillet yield was compared between the surubi and pacu species, in a completely randomized design (CRD) with two treatments and 10 replications. For the physical characteristics of the fillet used the CRD with three treatments and 10 replications, compared by Tukey test. The surubi fillet yield was higher than the one for pacu. Water loss after thawing was more pronounced in pirarucu when compared to surubi. Water retention capacity of pirarucu samples was significantly higher than those for surubi. The $\mathrm{pH}$ for the surubi fillet was $6.18 \pm$ 0.08 and for the pirarucu fillet was $6.27 \pm 0.15$, with no significant differences. The coloring of the surubi and pirarucu fillets are very similar due to the quality of feedd provided during captive production of these species of fish. The fish fillets for the species under study present characteristics of a succulent, soft and clear meat. It is important to emphasize the need for future tests on the acceptability and processing of this meat to stimulate consumption.
\end{abstract}

KEYWORDS: Fish. Native fish. Fish fillet.

\section{CARACTERIZACIÓN FÍSICA DE FILETES DE SURUBÍ (Pseudoplatystoma sp.), PACÚ (Piaractus mesopotamicus) Y PIRARUCÚ (Arapaimas gigas)}

RESUMEN: El objetivo de este estudio ha sido evaluar las características físicas del filete de surubí (Pseudoplatystoma sp.), pacú (Piaractus mesmopotamicus) y del pirarucú (Arapaimas gigas). Se adquirieron 10 ejemplares de surubí, pacú y lonchas de solomillo de Pirarucú. Se han medido los parámetros de rendimiento de la carcasa y las características físicas de los filetes como el color de la carne, capacidad de retención de agua, resistencia al corte y pérdida de líquido durante el cocimiento. Se ha comparado el rendimiento de los filetes entre las especies surubí y pacú en el delineamiento enteramente casualizado, con dos tratamientos y 10 repeticiones. A las características físicas del filete se han utilizado el delineamiento enteramente casualizado con tres tratamientos y 10 repeticiones, comparadas por el test de Tukey. El rendimiento del filete de surubí fue superior al de pacú. Las pérdidas de agua tras el descongelamiento fueron más pronunciadas en pirarucú en comparación al surubí. La capacidad de retención de agua de las muestras de pirarucú fue significativamente mayor que las del surubí. El pH

DOI: https://doi.org/10.25110/arqvet.v17i4.2014.5023

${ }^{1}$ Centro Universitário da Grande Dourados - UNIGRAN, Faculdade de Ciências Biológicas e da Saúde, Medicina Veterinária, Rua Balbina de Matos, 2121 Jd. Universitário CEP 79.824-900, Dourados-Mato Grosso do Sul, Brasil. clauciahonorato@yahoo.com.br 
del filete de surubí fue de $6.18 \pm 0.08$ y del filete de Pirarucú de $6,27 \pm 0.15$ y no presentaron diferencias significativas. La coloración del filete de surubí y del pirarucú es muy similar debido a la calidad de la alimentación proporcionada durante la producción de estas especies de peces en cautiverio. Los filetes de las especies de peces del estudio presentan características de carne suculenta, tierna y de coloración clara. Se destaca la necesidad de realización de futuros testes de aceptabilidad y de procesamiento de esta carne para estimular el consumo.

PALABRAS CLAVE: Pescado. Peces nativos. Filete de pescado.

\section{Introdução}

O mercado global de alimentos tem experimentado expansão sem precedentes e mudança nos padrões alimentares, tornando-se mais homogêneo e globalizado. Em 2013, as previsões do consumo de pescados aproximavam-se de 20 $\mathrm{kg}$ por habitante por ano, com produção estimada de mais de 160 milhões de toneladas (Food and Agriculture Organization of the United Nations, 2013). Além disso, a demanda por produtos à base de pescado deve aumentar nas próximas décadas, seja por razões socioeconômicas, de saúde ou religiosas. Essa tendência vem sendo observada e, atualmente, quase metade da produção de pescado já é originada da aquicultura. Assim, o aumento do consumo per capita de pescado será cada vez mais dependente da disponibilidade dos produtos da aquicultura e sua capacidade de adequação às exigências do mercado consumidor (ROCHA et al., 2013).

O pescado é a carne mais demandada mundialmente (SIDONIO et al., 2012) e a de maior valor de mercado. Porém, no Brasil, seu consumo ainda é baixo, mesmo tendo aumentado nos últimos anos para $11,17 \mathrm{~kg}$ por habitante por ano (BRASIL, 2013), valor ainda abaixo do mínimo recomendado pela Organização Mundial de Saúde, que é de 12 $\mathrm{kg}$ por habitante por ano (Food and Agriculture Organization of the United Nations, 2012), mas $14,5 \%$ a mais do que em relação ao ano anterior (BRASIL, 2010).

Apesar do baixo consumo de pescado pela população brasileira, a balança comercial deste produto encontra-se em déficit monetário e volume comercializado desde 2006 (BRASIL, 2011). Esses dados apontam que o consumo vem se expandindo, no entanto, parte das exigências do mercado não pode ser suprida por produtos nacionais devido à sazonalidade da produção (SIDONIO et al., 2012). Uma estratégia para suprir a demanda do mercado está na produção de espécies nativas com boa aceitação pelos consumidores, dentre as espécies de peixes destaca-se o surubim (Pseudoplatystoma sp.) e o pirarucu (Arapaimas gigas). Os dados da Secretaria de Aquicultura e Pesca em 2009 revelam que o Brasil produz um volume de 2126,7 toneladas de surubins por ano, sendo que a produção nos últimos cinco anos cresceu 84,53\% (BRASIL, 2009). Estudos como os realizados pelo SEBRAE na região norte do país de 2007 a 2010, demonstram tanto a viabilidade na área de produção quanto de mercado, bem como indicando as diversas dificuldades em se produzir o pirarucu.

Sua carne branca com pequena quantidade de espinhos intramusculares, sabor delicado e baixo teor de gordura, faz dessa espécie de água doce uma das mais apreciadas no Brasil (KUBITZA; ONO; CAMPOS, 2007). O mercado internacional principalmente o asiático tem demonstrado interesse crescente em aumentar o volume de compra dessas espécies de peixes, no entanto, ainda não se tem a caracterização deste produto. Apesar das lacunas ainda existentes no pacote tecnológico da produção do surubim, seu potencial produtivo associado à qualidade de sua carne desperta grande interesse para viabilizar sua produção. Nos últimos anos, a oferta se manteve bem inferior à demanda, sendo necessária a importação desses peixes de países vizinhos como o Paraguai, a Argentina, a Bolívia e a Colômbia (KUBITZA; ONO; CAMPO, 2007).

No entanto, uma das causas que apontam o baixo consumo de peixes está relacionada a falta de praticidade e de padronização do produto. Escassos são os estudos relacionados às características morfométricas, rendimento e composição de filé de peixes de água doce no Brasil, havendo poucos dados que permitam comparar as espécies, avaliar fatores críticos e visualizar o potencial de industrialização. Esses dados são imprescindíveis, pois fornecem subsídios às indústrias de processamento e aos piscicultores que podem estimar sua produção econômica (MACEDO-VIÉGAS; ROSSI, 2000; SOUZA, 2002). Outro ponto da cadeia de pescado está nas características físicas como a cor da carne dos peixes, que é um dos principais parâmetros avaliados pelos consumidores (KNOWLES et al., 2008). Além disso, a textura dos alimentos é um atributo de qualidade que envolve a percepção de características mecânicas (suculência, dureza, elasticidade, etc.), geométricas (tamanho) e de superfície. Entre as características relacionadas à textura, a dureza da carne é de fundamental importância para o consumidor e define o valor comercial do produto (JAIN; PATHARE; MANIKANTAN., 2007).

As características físicas são fatores primordiais para definir um produto de qualidade na mesa do consumidor, porém, nenhum desses parâmetros são conhecidos para estas espécies de peixes. Dessa forma, o objetivo deste estudo foi avaliar as características físicas de filé de surubim (Pseudoplatystoma sp.) pacu (Piaractus mesmopotamicus) e do pirarucu (Arapaimas gigas).

\section{Material e Métodos}

Foram adquiridos 10 exemplares das espécies surubim (Pseudoplatystoma sp.) e 10 de pacu (Piaractus mesopotamicus) e seis quilos de postas de filé de pirarucu (Arapaimas gigas) no comércio de Dourados/MS, no qual se certificou que estes eram provenientes de criação comercial e abatidos em frigorífico certificado por órgão de inspeção federal. Os peixes foram transportados em caixa térmica com gelo até o laboratório de análise de carne da Universidade Federal da Grande Dourados / UFGD. Esses foram pesados, decapitados e filetados (Comitê de ética em pesquisa - UNIGRAN - 292/10). Durante o processamento dos exemplares de surubim e pacu foram aferidos os seguintes parâmetros: peso total (PT); peso do filé (PF), comprimento padrão = aferido desde a ponta do focinho até o pedúnculo caudal (CP); altura corporal $=$ aferido desde a base da nadadeira dorsal até a parte ventral (AC); largura do filé (LF); comprimento do filé (CF); espessura do filé (EF). Todos os dados de ren- 
dimento foram calculados em porcentagem, em relação ao peso total. Para a análise do rendimento de carcaça, de filé e de resíduo de filetagem, foram utilizadas as seguintes equações: Porcentagem de Filé $(\% \mathrm{~F})=\mathrm{PF}$ x 100 / PT; Porcentagem de Carcaça $(\% \mathrm{C})=$ PCE x $100 /$ PT;

As características físicas dos filés das três espécies foram avaliadas quanto à cor da carne, capacidade de retenção de água, força de cisalhamento e perda de líquido durante o cozimento. Para essas análises foram realizadas 10 mensurações por filés.

Coloração da carne: As determinações da cor da carne foram realizadas utilizando-se um colorímetro (Konica Minolta $\left.{ }^{\circledR}\right)$, avaliando-se a luminosidade ( $\mathrm{L}^{*} 0=$ preto; 100 $=$ branco), a intensidade da cor vermelha $\left(\mathrm{a}^{*}\right)$ e a intensidade da cor amarela $\left(b^{*}\right)$.

Capacidade de retenção de água: Uma amostra de aproximadamente $2,0 \mathrm{~g}$ foi submetida à pressão de $10 \mathrm{~kg}$, durante cinco minutos, posteriormente pesado.

Perda de água na cocção: As amostras de filé foram assadas em forno a gás à temperatura de $175^{\circ} \mathrm{C}$, até atingirem $75^{\circ} \mathrm{C}$ no seu centro geométrico, mensurada por meio de um termômetro digital de sonda. Os pesos das amostras antes e depois da cocção foram utilizados para os cálculos das perdas totais.

Força de cisalhamento: As amostras de filés de surubim foram cortadas com três centímetros de diâmetro. Para a determinação da força de cisalhamento, utilizou-se aparelho Warner-BratzlerShear Force - mecânico com capacidade de 25 quilos e velocidade do seccionador de $20 \mathrm{~cm} / \mathrm{min}$.

O rendimento de filé foi comprado entre as espécies surubim e pacu em delineamento inteiramente casualizado (DIC) com dois tratamentos (espécies de peixes) e 10 repetições. As características físicas do filé utilizou-se o DIC com três tratamentos (espécies de peixes), com dez repetições. Quando foi encontrada diferença significativa $\mathrm{P}>0,05$ as médias dos tratamentos foram comparadas pelo teste de Tukey.

\section{Resultados e Discussão}

As espécies de peixes utilizadas são consideradas como excelentes pelo mercado consumidor, o surubim possui posição de destaque por ser uma carne saborosa de coloração branca que não possui espinhos em forma de $\mathrm{Y}$ em sua musculatura. No entanto, os peixes que possuem escamas são conhecidos como muito saborosos, mas apresentam grande quantidade de espinhos. Dentre os peixes de escamas que vem conquistando o mercado consumidor está o pirarucu, pelo sabor da sua carne e principalmente pelo seu tamanho de abate (90 a 120kg) o que possibilita a retirada dos espinhos intramusculares.

O rendimento de carcaça é um dos fatores primordiais para definir o rendimento do processamento do peixe. O rendimento de filé do surubim foi superior ao do pacu (Figura 1). O rendimento de filé do pacu pode estar sub estimado neste trabalho, pois um dos fatores determinantes deste índice é o tamanho do peixe para o abate. Basso e Ferreira (2011) estudando efeito do peso ao abate nos rendimentos dos processamentos do pacu (Piaractus mesopotamicus) descreveram que o rendimento de filé apresenta aumento linear conforme aumenta a classe de peso dos peixes. Outro fator que altera o rendimento de partes comestíveis do peixe está a relação entre as partes do corpo como tamanho de cabeça e nadadeiras. Este fator foi verificado por Faria et al. (2003), que estudaram os rendimentos da tilápia do Nilo e do pacu verificando que o pacu, por possuir menor cabeça, apresentou maior rendimento de carcaça e especialmente de filé.

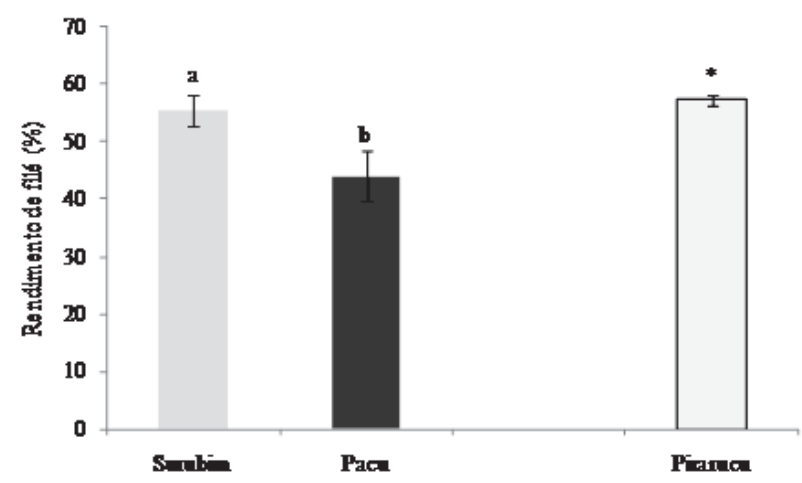

Figura 1: Rendimento de filé do pintado, pacu e pirarucu. (Letras distintas reportam diferença estatística pelo teste de Tukey $\mathrm{p}<0,05$ ). * Valor obtido em Carvalho et al. (2008).

Observa-se que o rendimento de filé das três espécies de peixes são superiores a $45 \%$. Esses resultados são semelhantes para algumas espécies de peixes relatadas por Santos, Gonzalez e Araujo (2001), que avaliaram rendimento de filé da traíra (Hoplias malabaricus) obtendo resultados de $48,63 \%$ para os machos e 46,12\% para as fêmeas. Marengoni, Souza e Caçador (1998) observaram rendimento de filé de 39,49\% do bagre-do-canal (Ictalurus puntactus). Para piracanjuba (Bryconorbignianus) foi observado rendimento de filé de 44,17\% (FREATO, 2005).

Os filés de surubim são comercializados inteiros devido ao tamanho e peso de abate do peixe em torno de um quilo, com peso do filé de $383,88 \pm 66,21 \mathrm{~g}$. O pirarucu é comercializado em postas de filé que corresponde a região mediana do filé, isso se deve ao peso de abate desta espécie de peixe que é em torno de $90 \mathrm{~kg}$, sendo a parte comestível denominada de manta. As postas de filés de pirarucu deste trabalho apresentaram 51,17 $\pm 15,39 \mathrm{~g}$.

As perdas de água após o descongelamento das amostras foram mais pronunciadas nas amostras de pirarucu em comparação ao surubim (Figura 2A). Apesar de não apresentar diferença estatística, esses resultados são reflexo da área de exposição das amostras, como o filé de pirarucu apresenta-se em pequenos pedaços, a perda de água é mais significativa para o meio externo. $\mathrm{O}$ fenômeno gaping (perdas de água por gotejamento) merece destaque, uma vez que é economicamente importante por dificultar o processamento e causar o desagregamento do filé. O gaping é originário na ruptura do tecido conjuntivo que causa a quebra dos blocos de miotomas (ROBB; KESTIN; WARRISS, 2000). Em pescado submetido a um estresse pré-abate são observadas modificações indesejáveis como maciez excessiva, o aumento na incidência de gaping e diminuição da capacidade de retenção de água (ERIKSON; SIGHOLT; SELAND1997; LAMBOOIJ et al., 2006). 
A

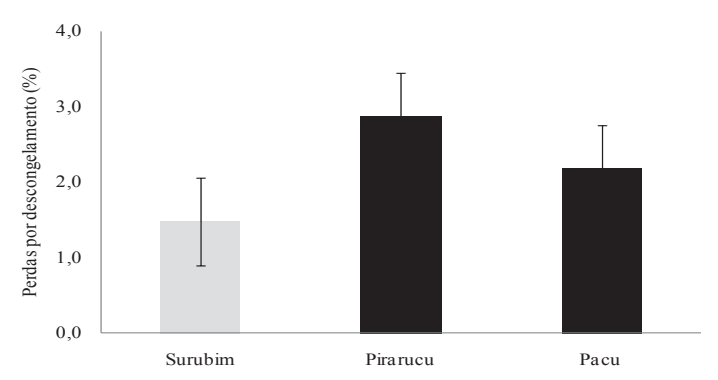

B

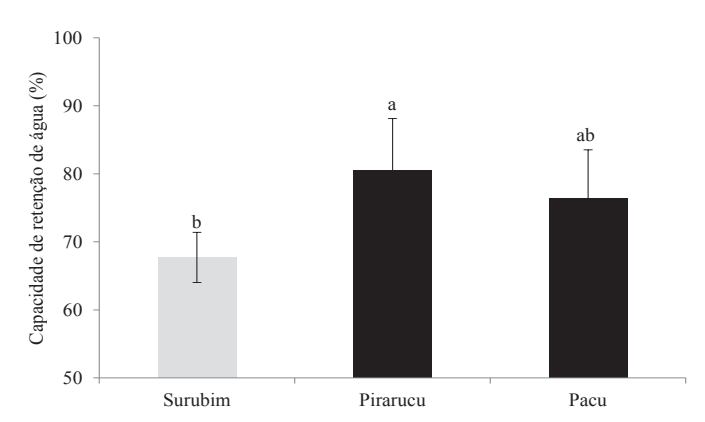

C

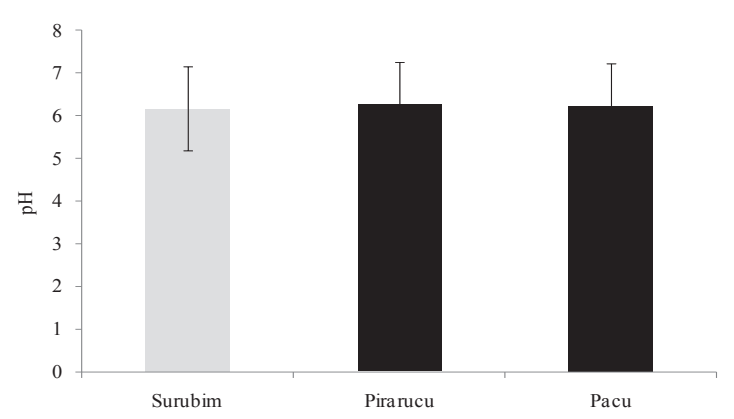

D

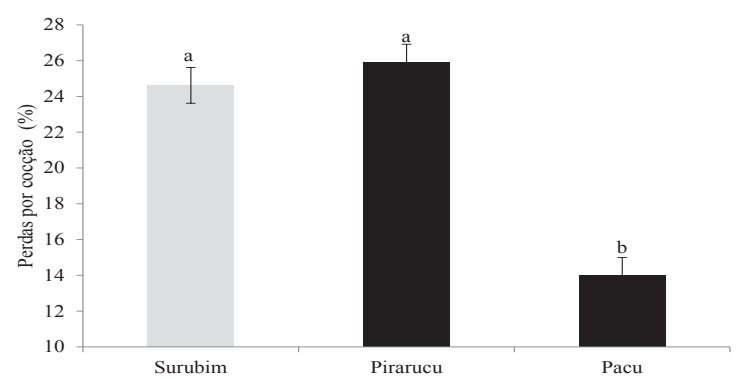

Figura 2: Comparação entre o filé de surubim e pirarucu de perdas por descongelamento (A), capacidade de retenção de água (B), $\mathrm{pH}$ (C) eperdas por cozimento (D). (Letras distintas reportam diferença estatística pelo teste de Tukey $\mathrm{p}<0,05)$

A capacidade de retenção de água das amostras de pirarucu foi significativamente superior as do surubim (Figura 2B). A capacidade de retenção de água é a habilidade da carne em reter a água contida em sua estrutura. Para o con- sumidor, essa característica está relacionada com o aspecto antes e durante a cocção e com a palatabilidade do produto final, e é dependente, entre outros fatores, do $\mathrm{pH}$, da força iônica e pressão osmótica (OLIVO, 2004).

$\mathrm{O} \mathrm{pH}$ do filé de surubim foi de $6,18 \pm 0,08$ e do filé de pirarucu de $6,27 \pm 0,15$ e não apresentaram diferenças significativas (Figura 2C). A escala de $\mathrm{pH}$ estende-se praticamente de 0 até 14 . Para a carne apenas um pequeno alcance de +/- pH 4,5 até pH 7 tem importância. Depois de atingir o valor de $\mathrm{pH}$ final, fica inalterado durante algum tempo, e no decurso da conservação aumenta um pouco (+/- 0,1 unidades pH) (SILVA; HONORATO, 2013). Em peixes, o valor de $\mathrm{pH}$ chega a 5,4-5,8 dentro de um período de 24 horas depois do abate (FONTES et al., 2007). Ressalta-se que esses valores de $\mathrm{pH}$ são de peixes recém-abatidos, e que são escassos os estudos de caracterização de filés em diferentes tempo de prateleira.

De forma geral, observa para a maioria das carnes uma relação entre aumento na capacidade de retenção de água e diminuição nas perdas por cocção (PINHO; KINDLEIN; MACMANUS, 2012). No entanto, nas espécies de peixes estudadas só foi observado esta correlação para o pirarucu. Observou-se que o pacu obteve a menor taxa de perdas por cocção. Essas perdas referem-se ao grau de encolhimento durante a cocção que estaria diretamente, correlacionado com a perda de suculência.

A coloração do filé do surubim e do pirarucu são muito semelhantes (Figura 3), isto se deve a qualidade da alimentação fornecida durante a produção em cativeiro dessas espécies de peixe. De uma forma geral, o mercado consumidor espera que a carne de peixe de água doce seja branca e luminosa. Ressalta-se que a cor da carne dos peixes é um dos principais parâmetros avaliados pelos consumidores (KNOWLES et al., 2008). Em turbot (Psetta máxima) ou carpa capim (Ctenopharyngodon idella) submetidos ao abate por água e gelo ou choque elétrico não foi observado diferença nos parâmetros de luminosidade ( $\left.\mathrm{L}^{*}\right)$, intensidade da cor vermelha-verde $\left(a^{*}\right)$ e intensidade da cor amarela-azul $\left(b^{*}\right)$ dos filés (SCHERER, 2005; KNOWLES et al., 2008). Igualmente, Roth, Slinde e Robb(2006) também não observaram variações na luminosidade $\left(\mathrm{L}^{*}\right)$ dos filés de salmões (Salmo salar) submetidos ao choque elétrico ou percussão craniana. Contrariamente, em carpa comum (Cyprinus carpio) submetida a narcose com $\mathrm{CO}_{2}$ foi observado maior valor de $\mathrm{L}^{*} \mathrm{e}$ menor de $\mathrm{a}^{*}$ nos filés em relação ao tratamento com anestésico, água e gelo e asfixia, respectivamente (RAHMANIFARAH; SHABANPOUR; SATTARI, 2011). O armazenamento do pescado em gelo pode causar mudanças na coloração do músculo. Em estudo com filés de meagre (Argyrosomus regius), armazenados em gelo durante 18 dias, foi observado aumento nos valores de $\mathrm{L}^{*}, \mathrm{~b}^{*} \mathrm{e}$ diminuição da dureza, mostrando as mudanças físicas que ocorrem durante a armazenagem em gelo (HERNANDEZ et al., 2009). 


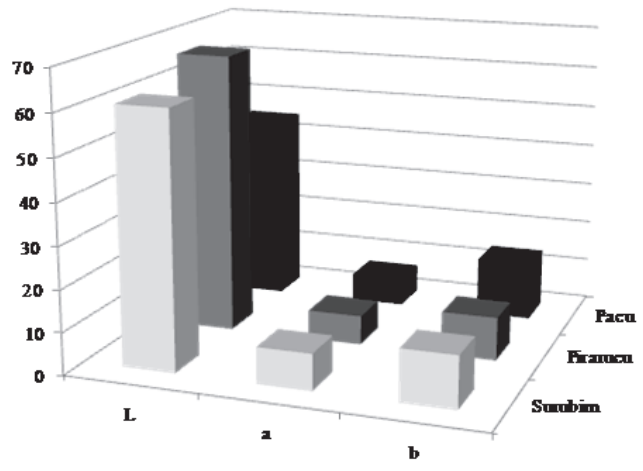

Figura 3: Comparação entre parâmetros de luminosidade $\left(\mathrm{L}^{*}\right)$, intensidade da cor vermelha-verde $\left(a^{*}\right)$ e intensidade da cor amarela-azul $\left(b^{*}\right)$ dos filés de Surubim, Pirarucu e Pacu.

Entre as espécies de peixes estudadas não foram observadas diferenças estatísticas no parâmetro força de cisalhamento (Figura 3). Ressalta-se que a força de cisalhamento dessas espécies de peixe estudadas são atribuídas a carnes extremamente macias. Traçando um comparativo entre as demais carnes consumidas no país, como para o contrafilé de bovinos da raça nelore observa-se força de cisalhamento de 6,7 kgf, sendo esta considerada como carne rígida (ABULARACH; ROCHA; FELÍCIO, 2008). Para cortes como picanha, contra filé e costela observaram-se valores de 1,87 ; 1,$94 ; 2,92 \mathrm{kgf}$, respectivamente (PINHO; KINDLEIN; MACMANUS, 2012). Para peito de frango obteve-se valores entre 1,91 a 2,23kgf (GARCIAet al., 2005).

No que tange a maciez da carne de peixe, esta está relacionada com a constituição das fibras musculares que compõem o tecido. Diferente dos demais vertebrados, em peixes os tipos de fibra musculares são separados em três compartimentos, sendo que as fibras vermelhas são superficiais e se estendem em uma camada subdermal (logo abaixo da pele)e no septo transverso, são separadas por fibras intermediárias e as fibras brancas as quais ocupam mais de $90 \%$ das fibras muscular. Outro fator determinante é a organização da musculatura estriada organizada em miosseptos (bainha de tecido conjuntivo que separam os miomeros), geralmente os miomeros se assemelham a letra $\mathrm{W}$ rotacionado em $90^{\circ}$ (LEITÃO; SILVA; PORTELLA, 2012) o que proporciona uma carne mole.

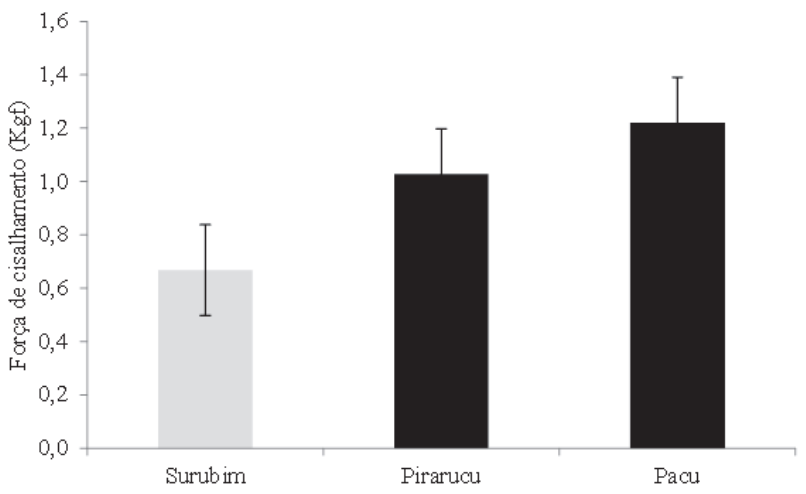

Figura 4: Força de cisalhamento dos filés de Surubim, Pirarucu e Pacu.

\section{Conclusão}

Os filés das espécies de peixe do estudo apresentam características de carne suculenta, macia e de coloração clara. Destaca-se a necessidade de realização de futuros testes de aceitabilidade e de processamento desta carne para estimular o consumo.

\section{Referências}

ABULARACH M. L. S.; ROCHA C. E.; FELÍCIO P. E. Características de qualidade do contra filé (m. L. dorsi) de touros jovens da raça Nelore. Ciência e Tecnologia de Alimentos, v.18, n.2, p.205-210, 2008.

BASSO, L.; FERREIRA, M. W. Efeito do peso ao abate nos rendimentos dos processamentos do pacu (Piaractus mesopotamicus). Revista Agrarian,v.4, n.12, p.134-139, 2011.

BRASIL, Ministério da pesca e aquicultura. Produção pesqueira e aquícola - 2008 e 2009. Disponível em: $<$ http://www.mpa.gov.br/\#info-estatstica/estatistica-dapesca-e-aquicultura $>$. Acesso em: 13 de fevereiro de 2014.

BRASIL. Ministério da Pesca e Aquicultura. Boletim estatístico da pesca e aquicultura 2011. Brasília: MPA, 2011. 60p.

BRASIL. Ministério da Pesca e Aquicultura. Boletim estatístico da pesca e aquicultura: Brasil, Brasília: MPA, 2010. 128p.

BRASIL. Ministério da Pesca e Aquicultura. Consumo de pescado no Brasil aumenta $23,7 \%$ em dois anos. 2013. Disponível em: <http://www.mpa.gov.br/index.php/ imprensa/noticias/2226-consumo-de-pescado-no-brasilaumenta-237-em-dois-anos>. Acesso em: 18 março 2014.

CARVALHO, S. E. Q. et al. Rendimento do file de pirarucu. Revista Brasileira de Engenharia de Pesca, v. 3, p. 21-22, 2008 .

ERIKSON, U.; SIGHOLT, T.; SELAND, A. Handling stress and water quality during live transportation and slaughter of Atlantic salmon (Salmosalar). Aquaculture, v. 149, p. 243-252, 1997.

FARIA R. H. S. A. et al. Rendimento do Processamento da tilápia do Nilo (Oreochromis niloticus Linnaeus) e do pacu (Piaractus mesopotamicus Holmberg,1887). Acta Scientiarum Animal Sciences, v. 25, n. 1, p. 21-24, 2003.

FONTES, M. C. et al. Estado de frescor e qualidade higiênica do pescado vendido numa cidade do interior de Portugal. Arquivo Brasileiro de Medicina Veterinária e Zootecnia, v. 59, n. 5, p. 1308-1315, 2007.

FOOD AND AGRICULTURE ORGANIZATION OF THE UNITED NATIONS. The state of world fisheries and aquaculture 2012. Rome: FAO, 2012. 209p. 
FREATO, T. A. Efeito do peso de abate nos rendimentos do processamento da piracanjuba (Brycon orbignyanus, VALENCIENNES, 1849). Ciência Agrotécnica, v. 29, n. 3, p. 676-682, 2005.

GARCIA, R. G. et al. Desempenho e qualidade da carne de frangos de corte alimentados com diferentes níveis de sorgo em substituição ao milho. Arquivos Brasileiros Medicina Veterinária e Zootecnia,v. 57, n. 5, p. 634-643, 2005.

HERNÁNDEZ, M. D. et al. 2009. Sensory, physical, chemical and microbiological changes in aquaculture meager (Argyrosomus regius) fillets during ice storage. Food Chemistry, v. 11, p. 237-245, 2009.

JAIN, D.; PATHARE, P. B; MANIKANTAN, M. R. Evaluation of texture parameters of Rohu fish (Labeorohita) during iced storage. Journal Food Engineer, v. 81, p. 336340, 2007.

KNOWLES, T. G. et al. Effect of electrical stunning at slaughter on the carcass, flesh and eating of farmed sea bass (Dicentrarchus labrax). Aquaculture Research, v. 38, p. 1732-1741, 2008.

KUBITZA, F.; ONO, E. A.; CAMPOS, J. L. Os caminhos da produção de peixes nativos no Brasil: uma análise da produção e obstáculos da piscicultura. Panorama da Aquicultura. v. 17, n. 102, p. 14-3, jul/ago, 2007.

LAMBOOIJ, E. et al. Electrical stunning followed by decapitation or chilling of African catfish (Clarias gariepinus): assessment of behavioral and neural parameters and product quality. Aquaculture Research, v. 37, p. 6170, 2006.

LEITÃO, N. J.; SILVA, M. D.; PORTELLA, M. C. Crescimento muscular em peixes: influência de fatores externos nas fases iniciais da criação. Panorama da Aquicultura, v. 129, 2012.

MACEDO-VIEGAS, E. M. M.; ROSSI, F. Técnicas de Processamento de Peixes. Viçosa-MG, 2000.

MARENGONI, N. G.; SOUZA, M. R.; CAÇADOR, W. C. Rendimento de filetagem de bagre africano (Clarias gariepinu) e bagre americano (Ictalurus punctatus). In: REUNIÃO ESPECIAL da SOCIEDADE BRASILEIRA PARA O PROGRESSO DA CIÊNCIA. Maringá, SBPC. Anais... p. 523-524, 1998.

OLIVIO, R. Atualidades na qualidade da carne de aves. Revista Nacional da Carne, v. 28, p. 38-50, 2004.

PINHO, A. P. S.; KINDLEIN, L.; MCMANUS, C. Lipídios totais, textura e perda por cocção de cortes de carne bovina de diferentes marcas comerciais. Acta Scientiae Veterinariae, v. 40, n. 3, p. 1-8, 2012.

RAHMANIFARAH, K.; SHABANPOUR, B.; SATTARI,

A. Effects of clove oil on behavior and flesh quality of common carp (Cyprinus carpio L.) in comparison with pre-slaughter CO2 stunning, chilling and asphyxia. Turk Journal Fish Aquatic Science, v. 11, p. 139-147, 2011.

ROBB, D. H. F.; KESTIN, S. C.; WARRISS, P. D. Muscle activity at slaughter: I. Changes in flesh colour and gaping in rainbow trout. Aquaculture, v. 182, p. 261-269, 2000.

ROCHA, C. M. C. et al. Avanços na pesquisa e no desenvolvimento da aquicultura brasileira. Pesquisa Agropecuária Brasileira, v. 48, n. 8, p. 4-6, 2013.

ROTH, B.; SLINDE, E.; ROBB, D. H. F. Field evaluation of live chilling with $\mathrm{CO} 2$ on stunning Atlantic salmon (Salmosalar) and the subsequent effect on quality. Aquaculture Research, v. 37, p. 799-804, 2006.

SANTOS, L. N.; GONZALEZ, A. F.; ARAUJO, F. G. Dieta do tucunaré-amarelo Cichla monoculus (Bloch \& Schneider) (Osteichthyes, Cichlidae), no Reservatório de Lajes, Rio de Janeiro, Brasil. Revista Brasileira de Zoologia, v. 18, p. 191-204, 2001.

SILVA, E. F.; HONORATO, C. A. Tecnologia de processamento de salga úmida e salga seca de filé com e sem pele de pacu (Piaractus mesopotamicus). Nucleus Animalium, v. 5, n. 1, p. 47-53, 2013

SCHERER, R. Effect of slaughter method on postmortem changes of grass carp (Ctenopharyngodon idella) stored in ice. Journal of FoodScience, v. 70, p. 348-354, 2005.

SIDONIO, L. et al. Panorama da aquicultura no Brasil: desafios e oportunidades. BNDES Setorial, v. 35, p. 421463, 2012.

SOUZA, M. L. R. Comparação de seis métodos de filetagem, em relação ao rendimento de filé e de subprodutos do processamento da Tilápia-do-Nilo (Oreochromis niloticus). Revista Brasileira de Zootecnia, v. 31, n. 3, p. 1076-1084, 2002.

Recebido em: 16.06 .2014 Aceito em: 29. 12.2014 